\title{
Single rod impact tests: Analytical, experimental, and modelling investigation
}

\author{
Yongchao Huang and Clive R Siviour ${ }^{\mathrm{a}}$ \\ Department of Engineering Science, University of Oxford, UK
}

\begin{abstract}
This work investigates the single rod impact test technique, from which wave propagation characteristics, i.e. the propagation coefficient and wave speed, can be obtained and used to identify the material modulus as a function of frequency. To accomplish this, an elastic Titanium rod and a viscoelastic Polymethyl Methacrylate (PMMA) rod have been tested. With a known modulus, the Titanium rod modulus was successfully recovered from the wave propagation coefficient in a frequency range of $5 \mathrm{kHz} \sim 30 \mathrm{kHz}$, which verifies the validity of this technique. The PMMA modulus identified by this technique agrees reasonably with the modulus derived from DMA tests in the range $2 \mathrm{kHz} \sim 20 \mathrm{kHz}$. The good agreement between the theoretical and simulation-based modulus further confirms the effectiveness of the single rod impact test in this use. It is also demonstrated that the single rod impact technique is capable of detecting the minor mechanical changes induced by temperature variations as small as $2^{\circ} \mathrm{C}$.
\end{abstract}

\section{Introduction}

A number of mechanical testing techniques have become available to obtain intrinsic material properties from low to high strain rates, these include classic tension, compression and torsion tests, drop weight test, SHPB test, Taylor and plate impact tests, etc. By looking at material behaviour at various loading speeds, some of these techniques directly yield material properties such as modulus, whist others present the stress-strain curves. However, classic testing methods have been developed for most materials but are confined to low strain rate testing and may pose specific requirements on the material shape being tested due to clamping arrangements. High rate testing methods such as SHPB have very limited capacity in testing soft materials such as rubber-like materials due to some un-resolved critical issues such as stress equilibrium in specimens. Impact tests on a single long rod, as performed earlier by Korsky [1], Bacon [2], and more recently Hillstrom [3] and Benatar [4], have been validated to be effective in deriving material properties such as modulus and Poisson's ratio. The experimental setup for a single rod impact test is much more simple and convenient compared with other material testing techniques, normally a long specimen rod is needed (otherwise wave separation techniques may be required), and any excitations, such as pendulum, air gun, or even hand, that can generate tiny disturbance in the bar are theoretically applicable, the monitored quantities can be either displacement, strain, or particle velocity, etc. By applying frequency domain analysis to the measured waves, wave propagation characteristics such as damping and wave speed can be easily obtained, and based on the propagation characteristics the frequency-dependent material properties, in particular the complex modulus,

\footnotetext{
${ }^{a}$ Corresponding author: clive.siviour@eng.ox.ac.uk
}

can be derived. Instead of directly measuring the material stress-strain behaviours under various loading conditions, this approach provides a different way to look at the frequency-dependent material behaviours. Although the frequency, another concept comparable to strain rate, that can be achieved from a single rod impact test is limited by a number of factors such as the experimental setup itself and the numerical analysis method, this technique has the potential to be improved for testing a wide range of materials such as metals and polymers.

\section{Theoretical basis}

The work is based on the wave propagation theories in a slender rod. Many theories have been established for characterizing wave propagation in a rod, either elastic [5] or viscoelastic [6]. These theories vary in whether it is $1 \mathrm{D}$ or $3 \mathrm{D}$, what cross-section it can be applied to, etc. The complexity of these theories is out of this paper's scope, and only basic wave propagation theories will be introduced here.

The time domain displacement and frequency domain strain expressions are normally employed as solutions to the wave equation of a propagating wave:

$$
\begin{aligned}
& u(x, t)=A e^{-\gamma x} e^{i \omega t}+B e^{+\gamma x} e^{i \omega t} \\
& \tilde{\varepsilon}(x, \omega)=\tilde{P}(\omega) e^{-\gamma x}+\tilde{N}(\omega) e^{\gamma x} .
\end{aligned}
$$

In which

$$
\gamma(\omega)=\alpha(\omega)+i \kappa(\omega), \quad(\alpha>0) .
$$

The functions A, B, $\tilde{P}, \tilde{N}$ are determined by initial conditions. $\gamma$ is termed the propagation coefficient, the attenuation coefficient $\alpha$ is a positive even function while 
the wavenumber $\kappa$ is an odd function (positive for $\omega>0$ ). The wavenumber $\kappa$ is associated with the wave speed by

$$
\kappa(\omega)=\frac{\omega}{c(\omega)} .
$$

Furthermore, the propagation coefficient $\gamma$ can be related to the complex modulus $E^{*}$ by

$$
\gamma^{2}(\omega)=\frac{-\rho \omega^{2}}{E^{*}(\omega)}
$$

Which further yields

$$
\begin{gathered}
E^{\prime}(\omega)=\frac{\rho \omega^{2}\left(\kappa^{2}-\alpha^{2}\right)}{\left(\kappa^{2}+\alpha^{2}\right)^{2}}, \quad E^{\prime \prime}(\omega)=\frac{\rho \omega^{2}(2 \kappa \alpha)}{\left(\kappa^{2}+\alpha^{2}\right)^{2}}, \\
\tan \delta=\frac{2}{\frac{\kappa}{\alpha}-\frac{\alpha}{\kappa}}
\end{gathered}
$$

or

$$
\begin{aligned}
\alpha^{2} & =\frac{\rho \omega^{2}}{2} * \frac{\sqrt{E^{\prime 2}+E^{\prime \prime 2}}-E^{\prime}}{E^{\prime 2}+E^{\prime 2}}, \\
\kappa^{2} & =\frac{\rho \omega^{2}}{2} * \frac{\sqrt{E^{\prime 2}+E^{\prime \prime 2}}+E^{\prime}}{E^{\prime 2}+E^{\prime \prime 2}} .
\end{aligned}
$$

Therefore, if either the modulus and/or the wave propagation coefficient is known, the other one can be calculated by using Eq. (6) or Eq. (8). In this paper, Eq. (6) is used in single rod impact test analysis while Eq. (8) is adopted in the DMA analysis. Note that Eq. (5), Eq. (6) and Eq. (8) are all 1D-based, thus appropriate revision may be required if $3 \mathrm{D}$ effects, such as geometric dispersion, are to be included.

Imagine a wave is propagating in an infinite rod, and 2 strain pulses are measured at 2 sections $x_{1}$ and $x_{2}$ without overlapping, then the 2 sub-coefficients can be obtained by

$$
\alpha=-\frac{\ln \left|\frac{\tilde{\varepsilon}\left(x_{1}, \omega\right)}{\tilde{\varepsilon}\left(x_{2}, \omega\right)}\right|}{x_{1}-x_{2}}, \quad \kappa=-\frac{\text { angle }\left[\begin{array}{l}
\tilde{\varepsilon}\left(x_{1}, \omega\right) \\
\tilde{\varepsilon}\left(x_{2}, \omega\right)
\end{array}\right]}{x_{1}-x_{2}} .
$$

Besides, based on the general strain solution Eq. (2), it is convenient to derive the general expressions for displacement, particle velocity, acceleration, and force in frequency domain:

$$
\begin{array}{r}
\tilde{u}(x, \omega)=\frac{1}{\gamma} \tilde{P}(\omega) e^{-\gamma x}+\frac{1}{\gamma} \tilde{N}(\omega) e^{\gamma x} \\
\tilde{\dot{u}}(x, \omega)=\frac{i \omega}{\gamma}\left[\tilde{P}(\omega) e^{-\gamma x}-\tilde{N}(\omega) e^{\gamma x}\right] \\
\tilde{a}(x, \omega)=\frac{\omega^{2}}{\gamma}\left[\tilde{P}(\omega) e^{-\gamma x}-\tilde{N}(\omega) e^{\gamma x}\right] \\
\tilde{F}(x, \omega)=\frac{\rho A \omega^{2}}{\gamma^{2}}\left[\tilde{P}(\omega) e^{-\gamma x}+\tilde{N}(\omega) e^{\gamma x}\right] .
\end{array}
$$

Thus the strain in Eq. (8) can be replaced by displacement, particle velocity, acceleration, or force if these quantities are to be measured instead.

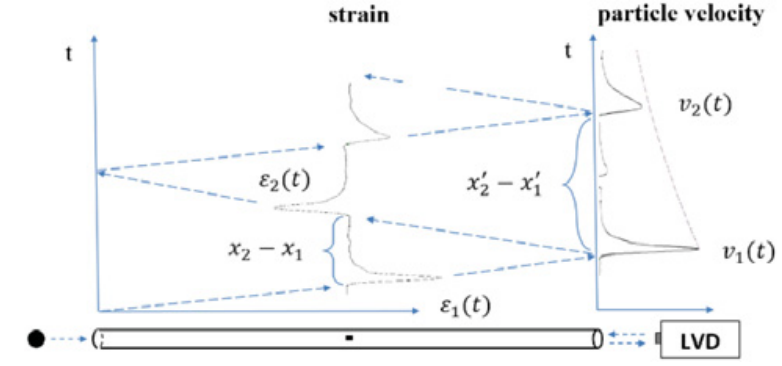

Figure 1. Wave propagation in a single rod impact test.

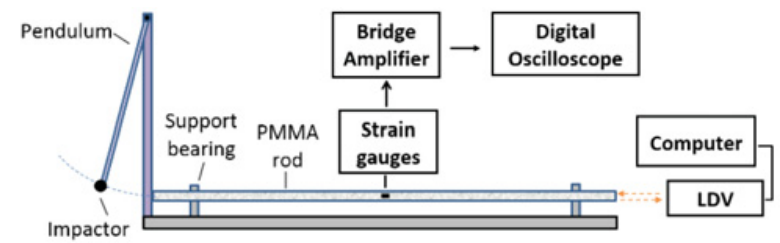

Figure 2. Experimental setup of the single rod impact test.

One assumption that was made in deriving the propagation coefficient $\gamma$ Eq. (8) is that the 2 pulses can be measured separately by using an "infinite" rod. In a real test, an "infinite" rod is impossible but approximations can be made. A relatively long linear rod (either elastic or viscoelastic), with both ends free after impact, can serve as an ideal approximation of an "infinite" rod because waves reflected at a linear free end either remain the same (e.g. particle velocity) or with sign inversed (e.g. strain). Figure 1 graphically shows the wave traveling path during an impact test, pulse duration should be controlled to avoid wave superposition at the gauge station. Also, the distance between 2 pulses sets a lower limit on the useful frequency that can be measured without overlapping.

Another assumption associated with modulus calculation (Eq. (6)) is 1D wave propagation in the rod. This excludes 3D effects such as the radial inertia-induced dispersion, and poses requirements on rod geometry. A "slender" rod is normally required for minimizing the $3 \mathrm{D}$ dispersion effect, in fact it has been reported that dispersion effects can be ignored as long as the rod diameter is at least 10 times less than the wavelength [7]. For a rod with a fixed diameter, this requirement in turn poses an upper limit on the useful frequency without appreciable dispersion. Though 3D solutions have been reported [6], it is our intension here that the dispersion effect be cancelled at the initial stage of data processing, this was achieved by applying Love's 3D theory [5] and adopting the phase-only dispersion correction methodology [8].

\section{Experimental implementation}

\subsection{Experimental setup}

The experimental setup of a single rod impact test is shown in Fig. 2. A portable pendulum setup, with the geometry of about $1.5 \times 0.1 \times 0.5 \mathrm{~m}(\mathrm{~L} \times \mathrm{W} \times \mathrm{H})$, was designed. The base is made of aluminium and the pendulum arm has a length of about $30 \mathrm{~cm} .2$ rods were used in the 
Table 1. Impactors and corresponding drop heights.

\begin{tabular}{|c|c|}
\hline Impactors & Drop heights (cm) \\
\hline$\varnothing 12.50 \mathrm{~mm}$ PMMA ball & $30,28,26,24,22,20,18$ \\
\hline$\varnothing 12.34 \mathrm{~mm}$ Aluminium ball & $27,25,23,21,19$ \\
\hline
\end{tabular}

pendulum impact tests: 1 PMMA rod with geometry of $\emptyset 13 \times 1000 \mathrm{~mm}$ and 1 Titanium rod of $\emptyset 12.7 \times 1000 \mathrm{~mm}$. They were supported by 2 ring bearings and are axially movable during the tests. The commercial PMMA rod has a mass density of $1180 \mathrm{~kg} / \mathrm{m}^{3}$, while the Titanium rod has a density of $4500 \mathrm{~kg} / \mathrm{m}^{3}$. Grease was used to reduce the friction at the ring bearings to avoid unexpected energy dissipation.

Two pairs of axially mounted strain gauges, each consists of 2 diametrically opposite strain gauges, were attached on the middle surface. A Laser Doppler Vibrometer (LDV) was made use of in the $23.4{ }^{\circ} \mathrm{C}$ PMMA rod impact tests to measure particle velocities at the non-impact end. The collected particle velocity signals were recorded separately by a computer, while strain signals were first amplified and then recorded by a digital oscilloscope.

Spherical balls are generally preferred in this impact tests as they can generate higher frequency components than cylinder impactors [2]. Also, as during wave propagation significant extension of pulse duration may appear as a result of the viscoelastic nature, the ball diameter is expected to be as small as possible. By taking these considerations into account, an impactor selection process was performed and after examining wave superposition in the recordings produced by different impactor types, finally 2 impactors made of different materials, i.e. the $\varnothing 12.50 \mathrm{~mm}$ PMMA ball and $\varnothing 12.34 \mathrm{~mm}$ Aluminium ball, were employed in our tests. And various drop heights (Table 1) have been adopted to generate different strain amplitudes within the linear viscoelastic range (LVR).

PMMA modulus can also be reported by Dynamic Mechanical Analysis (DMA). PMMA films, with the geometry of $1 \times 5 \times 30 \mathrm{~mm}$, were tested in tension mode; a frequency scan was performed at 5 frequencies $(1,5,10$, $25,40 \mathrm{~Hz}$ ) while temperature sweep was done at a ramping rate of $2{ }^{\circ} \mathrm{C} / \mathrm{min}$.

\subsection{Experimental results and discussions}

The $\varnothing 12.7 \times 1000 \mathrm{~mm}$ Titanium rod, with a known modulus of about $110 \mathrm{GPa}$, was tested at $T=22.6^{\circ} \mathrm{C}$ to verify the effectiveness of the single rod impact technique and the validity of the frequency domain analysis method. A typical Titanium rod impact strain recording is shown in Fig. 3, the nearly constant amplitudes indicate that no energy dissipation appeared during the strain wave propagation in the elastic Titanium rod.

The resulting wave propagation coefficient, wave speed, modulus and loss factor of the Titanium rod are presented in Fig. 4. It is observed that different drop heights yield almost the same results, with the attenuation coefficient, loss modulus and loss factor being zero, while

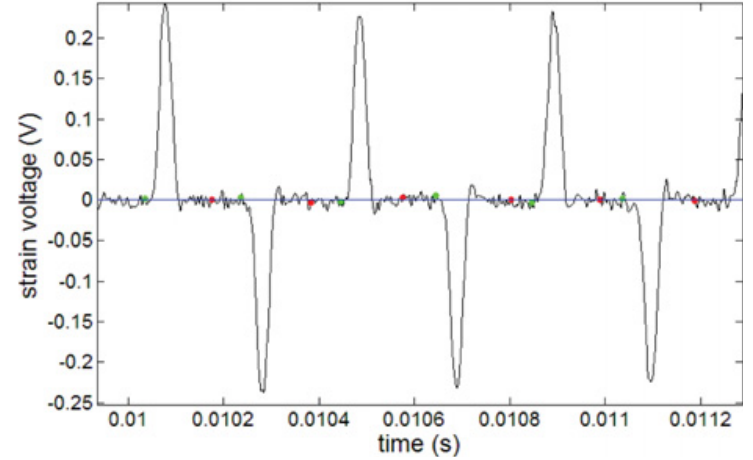

Figure 3. Strain waves in a Titanium rod impact test.

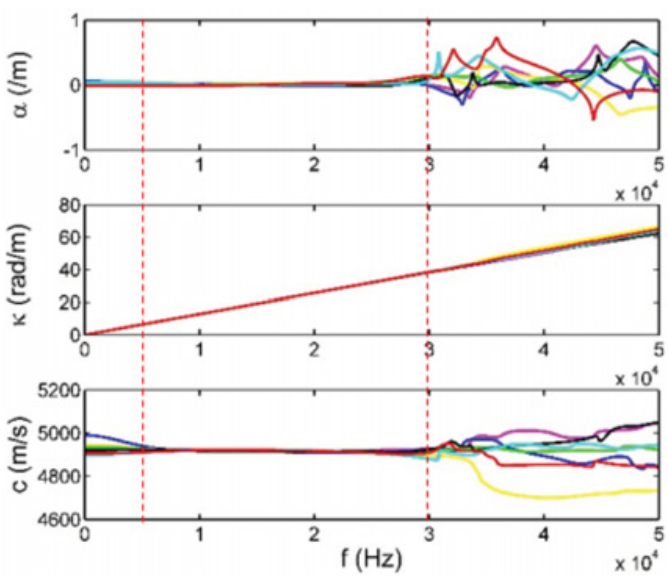

(a) Propagation coefficient and wave speed

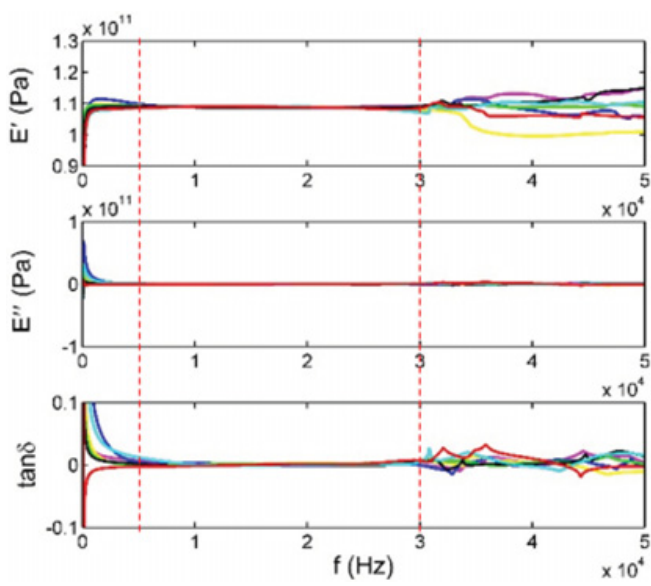

(b) Modulus and loss factor

$h=30 \mathrm{~cm}-\mathrm{h}=28 \mathrm{~cm} \quad \mathrm{~h}=26 \mathrm{~cm}-\mathrm{h}=24 \mathrm{~cm}-\mathrm{h}=22 \mathrm{~cm}-\mathrm{h}=20 \mathrm{~cm}-\mathrm{h}=18 \mathrm{~cm}$

Figure 4. Titanium rod impact results $\left(T=22 \cdot 6^{\circ} \mathrm{C}\right)$.

the storage modulus being about $110 \mathrm{GPa}$, which yields a wave speed of about $4944 \mathrm{~m} / \mathrm{s}$. The experiment-derived modulus agree well with the theoretical one in a frequency range of $5 \mathrm{kHz} \sim 30 \mathrm{kHz}$, which effectively verifies the validity of this technique. The lower useful frequency limit is affected by the distance between 2 adjacent pulses, while the highest useful frequency might be limited by dispersion effects and the excitation. 


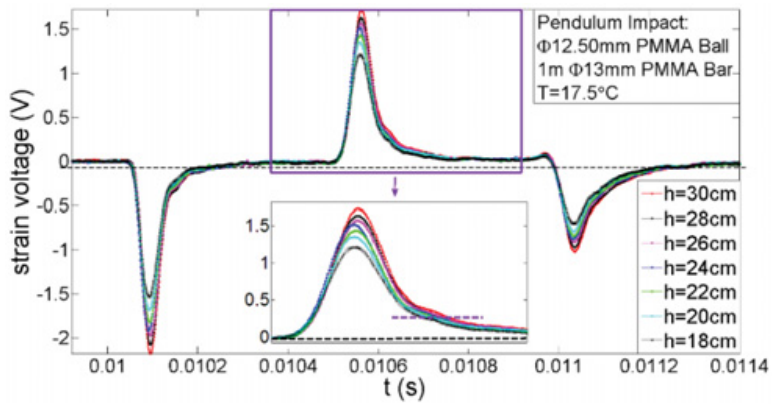

Figure 5. Strain recordings from PMMA rod impact tests.
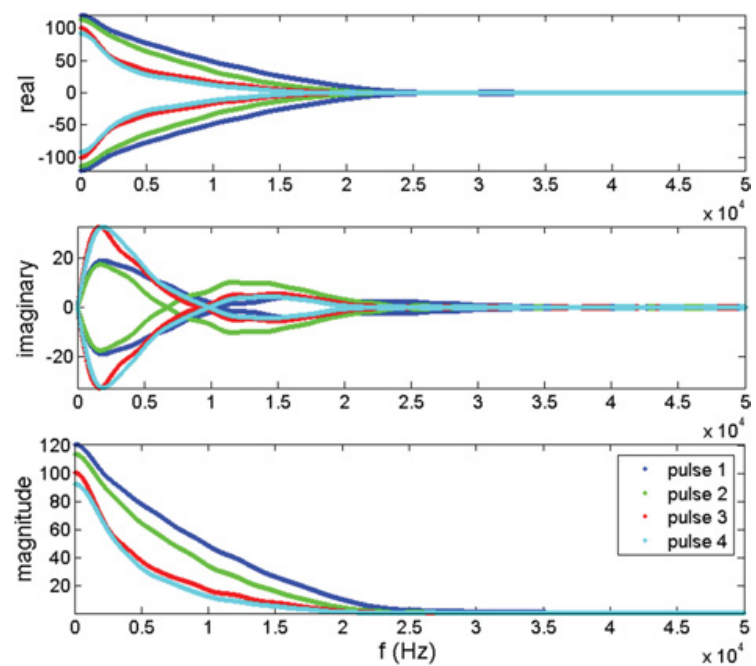

Figure 6. Fourier transform of the first 4 pulses of a PMMA rod impact signal.

PMMA rod impact tests were performed at 4 temperatures: $17.5^{\circ} \mathrm{C}, 19.5^{\circ} \mathrm{C}, 23.4^{\circ} \mathrm{C}, 27^{\circ} \mathrm{C}$. Figure 5 shows an example of some typical signals generated by different drop heights, and shown in Fig. 6 is an example of the Fourier transform of the collected signal, attenuation can be clearly seen in the magnitudes of the propagating pulses after Fourier transform.

Figure 7 presents the results obtained at these 4 temperatures. It can be seen that, the damping characteristics such as the attenuation coefficient, loss modulus and loss factor are observed to increase with temperature, while an inverse trend can be seen in the storage modulus and wave speed. This complies with our common sense that most materials tend to be more solid-like and become stiffer as temperature decreases, and stress waves propagates faster in solids than in soft medium. Evidence from molecular analysis also help support this phenomenon: molecular chains become more active and more space become available for mobility when temperature arises. It is thus concluded that this technique is sensitive enough for capturing the small variations in mechanical behaviours.

A comparison of the results derived from different drop heights confirms the independence of the wave propagation coefficient and modulus on strain amplitudes within the $\operatorname{LVR}(\varepsilon \leq 0.1 \%)$. Also consistent
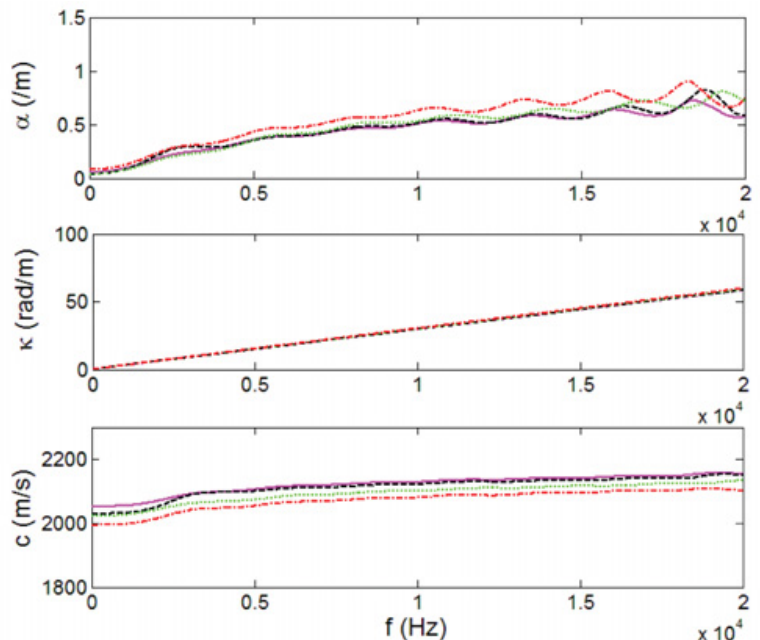

(a) Propagation coefficient and wave speed
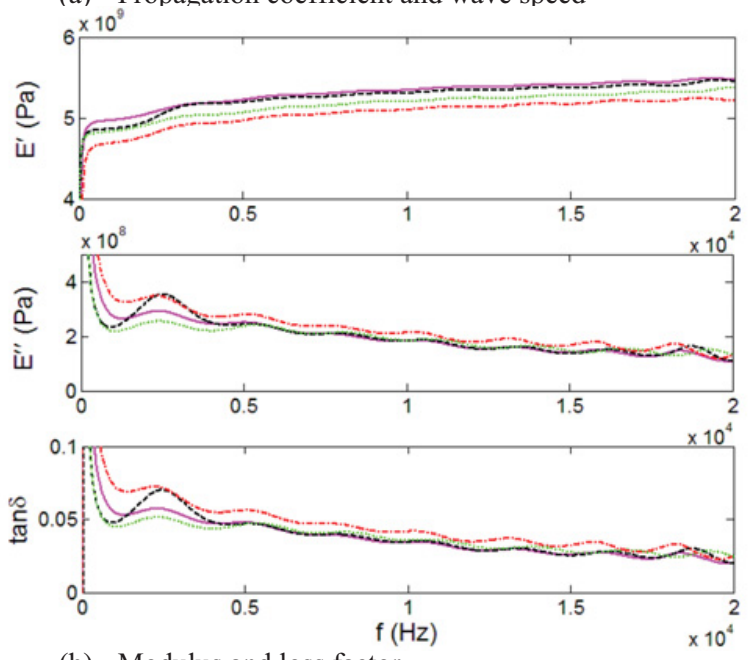

(b) Modulus and loss factor

$-\mathrm{T}=17.5^{\circ} \mathrm{C}-\mathrm{T}=19.5^{\circ} \mathrm{C}-\mathrm{T}=23.4^{\circ} \mathrm{C}-\mathrm{T}=27^{\circ} \mathrm{C}$

Figure 7. PMMA rod impact results $(\varnothing 12.34 \mathrm{~mm}$ Aluminium ball).

are the results produced by the 2 impactors as no major difference has been found between these results. Small deviations occur when comparing the strain-based and velocity-based attenuation coefficients (Fig. 8): the velocity measurements yield larger damping than strain measurements. The deviation in attenuation coefficient leads to a visible gap in loss modulus and loss factor but not in storage modulus, as storage modulus is less sensitive to the variation in attenuation coefficient than the other two. Noted that the useful frequency range for identifying this PMMA rod properties by using the single rod technique is $2 \mathrm{kHz} \sim 20 \mathrm{kHz}$.

The DMA-reported modulus are both temperaturedependent and frequency-dependent. By using both manual shifting and the formula-based Arrhenius shifting methods, 2 master curves were constructed at the reference temperature $T=23.4^{\circ} \mathrm{C}$. Comparison between the single rod impact results and DMA test results was made in Fig. 9. Deviations can be seen in wave speed and storage modulus while the damping characteristics $\left(\alpha, E^{\prime \prime}\right.$ and $\tan \delta$ ) show less variations. 

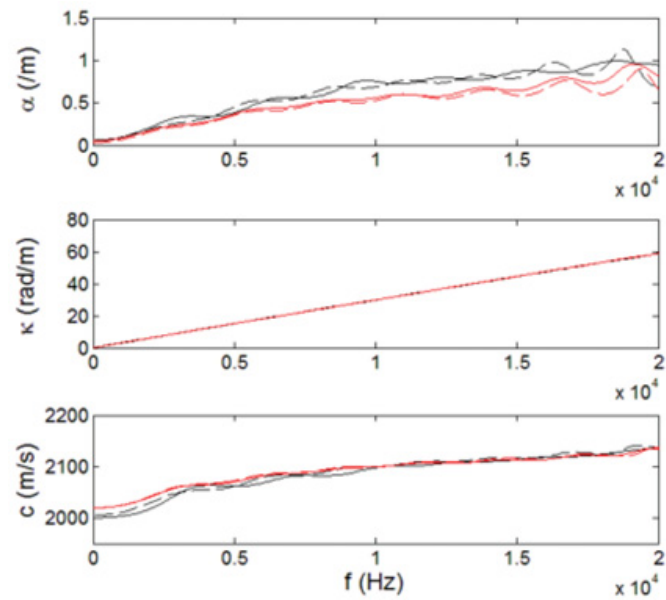

(a) Propagation coefficient and wave speed
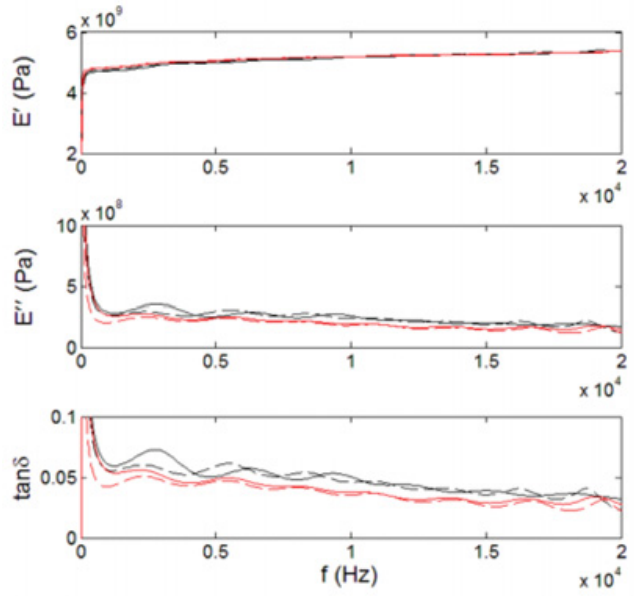

(b) Modulus and loss factor

Black: laser, red: strain gauge;

Solid: PMMA ball, dashed: aluminium ball

Figure 8. Comparison of results by 2 measurement methods $\left(T=23.4^{\circ} \mathrm{C}\right)$.

\section{Simulation investigation}

\subsection{Model definition}

Viscoelastic impact behaviours were simulated using the time domain PRONY model in ABAQUS/Explicit. The PRONY model is a parallel network model consisting of a group of springs and dashpots. It was defined by directly specifying the PRONY constants $\bar{g}_{i}^{P}, \bar{k}_{i}^{P}$ and $\tau_{i}$. These dominating constants, as shown in Table 2, were obtained by fitting the complex modulus from the $T=$ $23.4^{\circ} \mathrm{C}$ PMMA rod impact tests in the frequency range $2 \mathrm{kHz} \sim 30 \mathrm{kHz}$. Note the shear and bulk behaviours were defined separately in ABAQUS.

For the 2-term PRONY model, the frequencydependent theoretical modulus of are formulated as:

$$
\begin{aligned}
& E^{\prime}(\omega)=E_{0}\left(1-\sum_{i=12}^{2} \bar{g}_{i}^{p}\right)+E_{0} \sum_{i=1}^{2} \frac{\bar{g}_{i}^{p} \tau_{i}^{2} \omega^{2}}{1+\tau_{i}^{2} \omega^{2}} \\
& E^{\prime}(\omega)=E_{0} \sum_{i=1}^{2} \frac{\bar{g}_{i}^{p} \tau_{i} \omega}{1+\tau_{i}^{2} \omega^{2}} .
\end{aligned}
$$
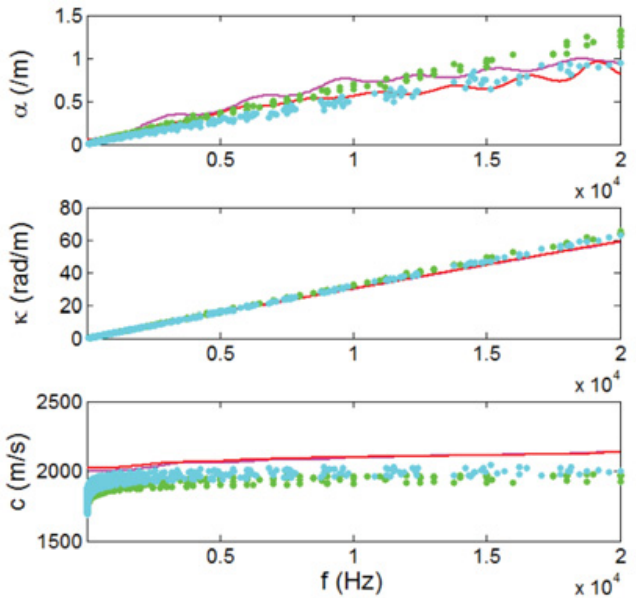

(a) Propagation coefficient and wave speed
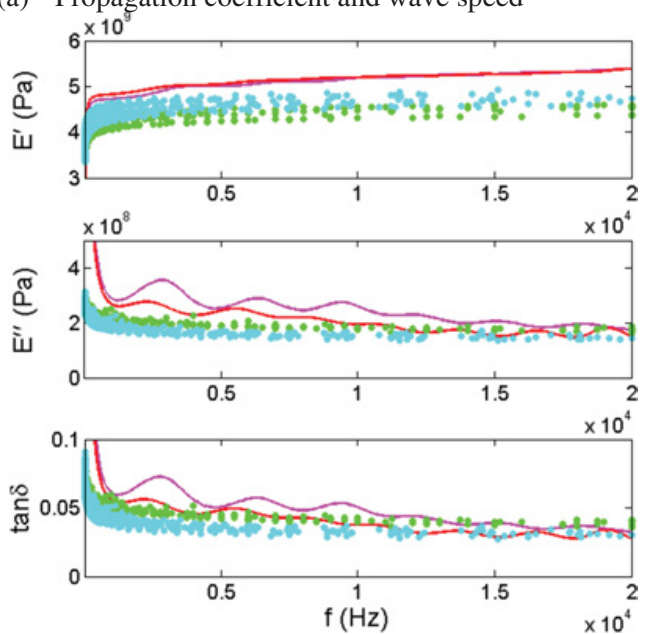

(b) Modulus and loss factor

$$
\begin{array}{|c|}
\hline \text { - laser (pmma ball) } \\
\hline \text { strain gauge (pmma ball) } \\
\text { DMA (manual shifting) } \\
\text { DMA (Arrhenius shifting) }
\end{array}
$$

Figure 9. Comparison of DMA and single rod impact results $\left(T=23.4^{\circ} \mathrm{C}\right)$.

Table 2. PRONY model constants.

\begin{tabular}{|c|c|c|}
\hline $\bar{g}_{i}^{p}$ & $\bar{k}_{i}^{p}$ & $\boldsymbol{\tau}_{i}(\mathbf{s})$ \\
\hline 0.031379 & 0 & $5.88 \mathrm{e}-006$ \\
\hline 0.092126 & 0 & $5.292 \mathrm{e}-005$ \\
\hline 0 & 0.031379 & $5.88 \mathrm{e}-006$ \\
\hline 0 & 0.092126 & $5.292 \mathrm{e}-005$ \\
\hline
\end{tabular}

Where $E_{0}$ is the instantaneous modulus, $\bar{g}_{i}^{p}$ and $\tau_{i}$ are the PRONY model constants specified in Table 2.

The $1^{\text {st }}$ pulse in the $T=23.4^{\circ} \mathrm{C}$ recordings was employed as the simulation input. In real case the deformation becomes uniform across the across-section in a distance longer than the largest cross-sectional dimension according to the Saint-Venant's principle, it is thus reasonable to distribute the input loading uniformly on the impact end cross-section.

\subsection{Simulation results and discussions}

The strain and particle velocity outputs are shown in Fig. 10 and the resulting wave propagation coefficient, wave speed, modulus and loss factor presented in Fig. 11, 


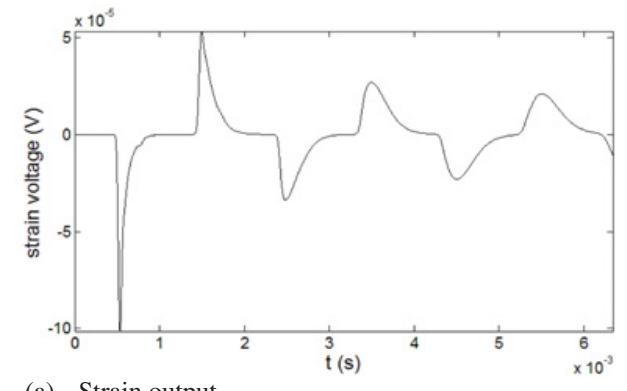

(a) Strain output

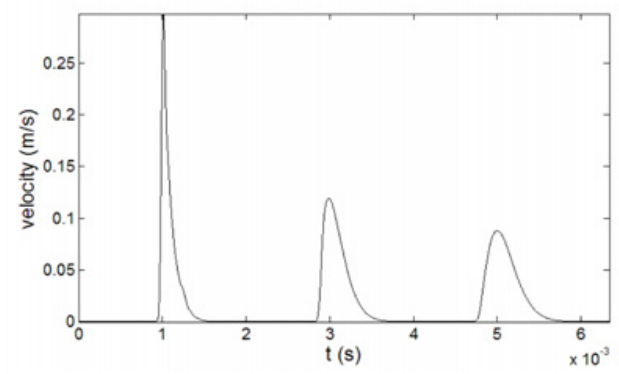

(b) Velocity output

Figure 10. Simulation signals.

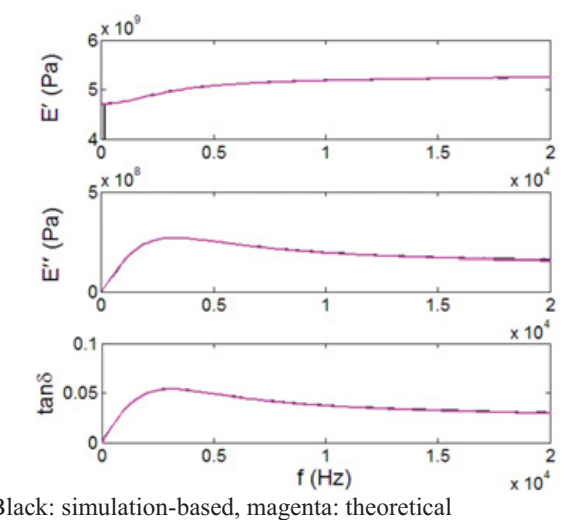

Figure 11. Comparison of the simulation-based and theoretical modulus.

accompanied by the theoretical modulus derived from Eq. (9). It can be seen that the simulation-derived moduli agree well with the theoretically predicted ones.

\section{Conclusions}

The ability of the single rod impact technique to derive the wave propagation characteristics and identify the material modulus was demonstrated in this paper. The Titanium rod moduli was recovered in a frequency range of $5 \mathrm{kHz} \sim 30 \mathrm{kHz}$ while the PMMA rod moduli were identified in the range $2 \mathrm{kHz} \sim 20 \mathrm{kHz}$. Temperaturecontrol tests also show that the single rod impact test technique is capable of capturing small changes in material mechanical properties induced by temperature variations as small as $2{ }^{\circ} \mathrm{C}$. The good agreement between the theoretical and simulation results further confirms the effectiveness of this technique and the validity of the frequency domain analysis method adopted.

Effort sponsored by the Air Force Office of Scientific Research, Air Force Material Command, USAF, under grant number FA8655-12-1-2015. The U.S Government is authorized to reproduce and distribute reprints for Governmental purpose notwithstanding any copyright notation thereon. The authors thank S Fuller of AFOSR and M Snyder and R Pollak of EOARD for their support.

\section{References}

[1] H. Korsky, Phil. Mag 8, 1 (1956)

[2] C. Bacon, Exp Mech 38 (1998)

[3] L. Hillstrom, M. Mossberg, B. Lundberg, J. Sound Vib 3, 230 (2000)

[4] A. Benatar, D. Rittel, A.L Yarin, J. Mech Phys Solids $51(2003)$

[5] A.E.H. Love, A treatise on the mathematical theory of elasticity (4th Edition, Cambridge University Press, 1927)

[6] H. Zhao, G. Gary, J. Mech Phys Solids 43 (1995)

[7] R.M. Davies, Phil. Trans. A, 240, 375 (1948)

[8] D.A. Gorham, J. Phys. E: Sci. Instrum., 16 (1983) 